CRYSTALLOGRAPHIC COMMUNICATIONS

ISSN 2056-9890

Received 10 July 2018

Accepted 16 August 2018

Edited by H. Ishida, Okayama University, Japan

Keywords: crystal structure; naphthoquinones; 2-hydroxy-3-(prop-2-yn-1-yl)naphthalene-1,4dione.

CCDC reference: 1862442

Supporting information: this article has supporting information at journals.iucr.org/e

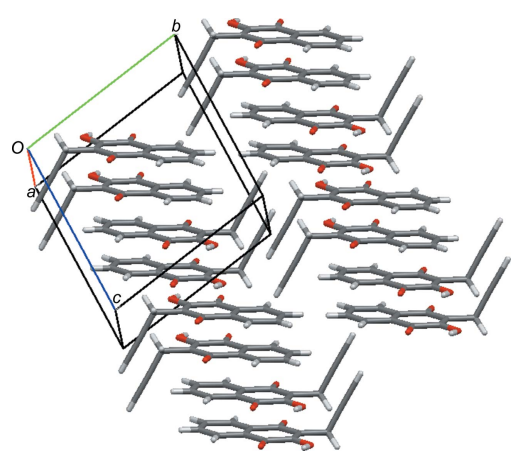

OPEN $\odot$ ACCESS

\section{Crystal structure of 2-hydroxy-3-(prop-2-yn-1-yl)- naphthalene-1,4-dione}

\author{
Isidório Raquel Geralda, ${ }^{\mathrm{a} *}$ Ottoni Flaviano Melo, ${ }^{\mathrm{a}}$ Alves Ricardo José ${ }^{\mathrm{a}}$ and Speziali \\ Nivaldo Lúcio $^{\text {b }}$
}

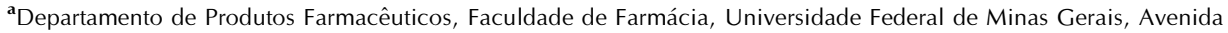
Antônio Carlos, 6627, Belo Horizonte Minas Gerais, CEP 31.270-901, Brazil, and ${ }^{\mathbf{b}}$ Departamento de Física, Instituto de Ciências Exatas, Universidade Federal de Minas Gerais, Avenida Antônio Carlos, 6627, Belo Horizonte, Minas Gerais, CEP 31.270-901, Brazil. *Correspondence e-mail: raquelgeisi@hotmail.com3" locator="is5498sup4.pdf">

The naphthoquinone unit of the title compound, $\mathrm{C}_{13} \mathrm{H}_{8} \mathrm{O}_{3}$, is essentially planar, with an r.m.s. deviation of $0.013 \AA$ for the non- $\mathrm{H}$ atoms. The essentially linear propargyl group is tilted by $c a 113^{\circ}$ relative to the naphthoquinone plane. In the crystal, molecules are linked via a pair of $\mathrm{O}-\mathrm{H} \cdots \mathrm{O}$ hydrogen bonds, forming an inversion dimer. The dimers are further linked via pairs of $\mathrm{C}-\mathrm{H} \cdots \mathrm{O}$ hydrogen bonds into a tape structure along [201] . No $\pi-\pi$ stacking is observed in the present case as it could be expected for naphthoquinone derivatives.

\section{Chemical context}

Lawsone (2-hydroxynaphtalene-1,4-dione), 1, shows promising in the synthesis of analogues of atovaquone, $\mathbf{2}$, an antimalarial drug (Nixon et al., 2013) also used in immunosuppressed patients affected by pneumonia caused by Pneumocystis carinii (Cirioni et al., 1995; Comley et al., 1995). Recent studies have shown that it can be also useful in the fight against cancer (Fiorillo et al., 2016; Ashton et al., 2016). Thus far unknown, 2-hydroxy-3-(prop-2-yn-1-yl)naphthalene1,4-dione (3) was obtained in a two steps one-pot procedure by reacting $\mathbf{1}$ with propargyl iodide, prepared in situ from propargyl bromide and potassium iodide. It opens the possibility for the synthesis of triazoles at the $\mathrm{C} 3$ position of $\mathbf{1}$ by $[2+3]$ alkyne-azide 1,3-dipolar cycloaddition enabling the preparation of 3-substituted lawsone derivatives with potential pharmacological activity, including atovaquone (2) analogues.

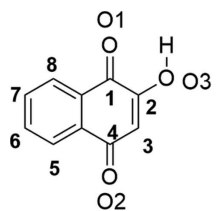

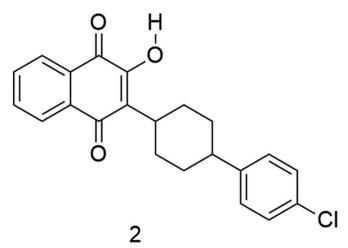

2<smiles>C=CCC1=CC(=O)c2ccccc2C1=O</smiles>

3
Treatment of $\mathbf{1}$ with a base leads to the formation of the corresponding enolate that can be $O$ - or $C$-alkylated depending on the nature of the counter-ion, reaction conditions and nature of the alkyl electrophile (Jordão et al., 2015). When 1 was reacted with propargyl bromide and sodium carbonate in DMF the 2-O-propargyl derivative was obtained in $20 \%$ yield (Valença et al., 2017). The 3-C-propargyl derivative had not been described thus far. In view of the importance of acetylenic compounds for [2 + 3] alkyne-azide 1,3- 


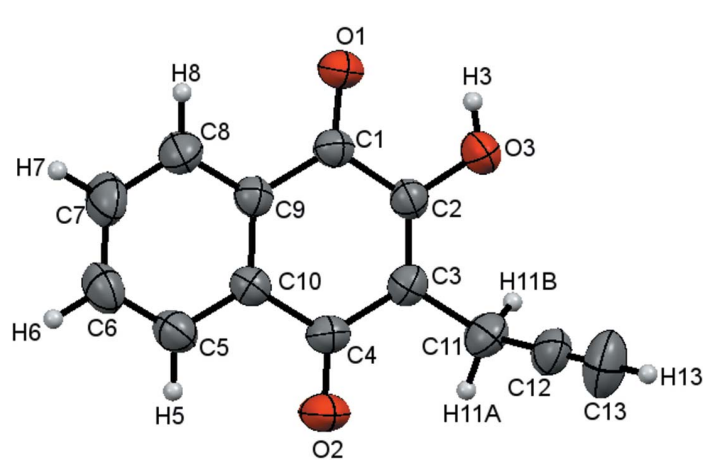

Figure 1

The molecular structure of the title compound 3. Displacement ellipsoids are drawn at the $50 \%$ probability level.

dipolar cycloaddition reactions, known as the click reaction, we decided to investigate the 2-O-versus 3-C-propargylation of 1. The 3-C-propargyl derivative is considered to be an interesting intermediate for the synthesis of 3-triazolo analogues of atovaquone, $\mathbf{2}$, and other bioactive 1,4naphthoquinones. After evaluating the influence of organic and inorganic bases, protic and aprotic solvents, alkylating agents, temperature and reaction time, we obtained 3 in $28 \%$ yield. No product of $O$-alkylation was observed in the reaction mixture.

\section{Structural commentary}

The molecular structure of the title compound, $\mathbf{3}$, is shown in Fig. 1. The naphthoquinone unit is essentially planar, with an r.m.s. deviation of $0.013 \AA$ for the non- $\mathrm{H}$ atoms. The $\mathrm{C}-\mathrm{O}$ bond lengths $[\mathrm{C} 1-\mathrm{O} 1=1.2217(18) \AA, \quad \mathrm{C} 2-\mathrm{O} 3=$ $1.3412(18) \AA$ and $\mathrm{C} 4-\mathrm{O} 2=1.2488(19) \AA]$ confirm the presence of 2-hydroxynaphthalene-1,4-dione in the crystalline state and are in agreement with the lengths found by Dekkers et al. (1996). The ${ }^{1} \mathrm{H}$ and ${ }^{13} \mathrm{C}$ NMR spectra and HMBC experiments confirm atoms $\mathrm{C} 1$ and $\mathrm{C} 4$ as carbonyls, as well as a hydroxy group at $\mathrm{C} 2$. The propargyl group adopts a nearly perpendicular position $\left[\mathrm{C} 3-\mathrm{C} 11-\mathrm{C} 12=112.70(14)^{\circ}\right]$ regarding the naphthalene ring system to avoid hindrance with the $\mathrm{O} 2$ and $\mathrm{O} 3$ atoms. The naphthoquinone ring system is characterized by the torsion angles $\mathrm{C} 4-\mathrm{C} 3-\mathrm{C} 11-\mathrm{C} 12=$ $-100.96(19)^{\circ}$ and $\mathrm{C} 2-\mathrm{C} 3-\mathrm{C} 11-\mathrm{C} 12=79.9(2)^{\circ}$.

\section{Supramolecular features}

In the crystal, $\mathrm{O}-\mathrm{H} \cdots \mathrm{O}$ and $\mathrm{C}-\mathrm{H} \cdots \mathrm{O}$ hydrogen bonds $\left(\mathrm{O} 3-\mathrm{H} 3 \cdots \mathrm{O} 1^{\mathrm{i}}\right.$ and $\mathrm{C} 5-\mathrm{H} 5 \cdots \mathrm{O} 2^{\mathrm{ii}}$; symmetry codes as in Table 1) are responsible for an infinite tape structure running along [201] . All the naphthoquinone units are arranged in a parallel manner with respect to each other, as shown in Fig. 2. $\pi-\pi$ stacking interactions are expected for naphthoquinone derivatives (Meyer et al., 2003). However, this type of interaction is not observed here, probably because of the C3 propargyl substituent.
Table 1

Hydrogen-bond geometry $\left(\AA{ }^{\circ}\right)$.

\begin{tabular}{lllll}
\hline$D-\mathrm{H} \cdots A$ & $D-\mathrm{H}$ & $\mathrm{H} \cdots A$ & $D \cdots A$ & $D-\mathrm{H} \cdots A$ \\
\hline $\mathrm{O} 3-\mathrm{H} 3 \cdots \mathrm{O} 1^{\mathrm{i}}$ & $0.89(3)$ & $2.06(3)$ & $2.8118(19)$ & $142(3)$ \\
$\mathrm{C} 5-\mathrm{H} 5 \cdots \mathrm{O} 2^{\mathrm{ii}}$ & 0.93 & 2.49 & $3.231(2)$ & 137 \\
\hline
\end{tabular}

Symmetry codes: (i) $-x+2,-y+1,-z$; (ii) $-x,-y+1,-z+1$.

\section{Database survey}

A search of the Cambridge Structural Database (CSD, Version 5.39, last update May 2018; Groom et al., 2016) for 2-hydroxy-naphthalene-1,4-dione revealed 40 structures and approximately 787 structures which possess the naphthalene1,4-dione moiety. 2-Hydroxy-3-(3-oxobutyl)naphthalene-1,4dione (Nasiri et al., 2006) and 2-hydroxy-3-(methyl-prop-1-en1-yl)naphthalene-1,4-dione (Alcantara Emiliano et al., 2016), compounds with structural similarity to the title compound, were also found. These compounds present a group linked to C3 with an angle nearly perpendicular to the naphthoquinone ring.

\section{Synthesis and crystallization}

The synthetic scheme is shown in Fig. 3. A mixture of propargyl bromide $(0.75 \mathrm{ml}, 4.47 \mathrm{mmol})$ and sodium iodide $(1.30 \mathrm{~g}, 5.33 \mathrm{mmol})$ in dry acetone $(3.5 \mathrm{ml})$ was stirred for $30 \mathrm{~min}$ at room temperature in a closed system. Then, a solution of lawsone $(0.1 \mathrm{~g}, 2.4 \mathrm{mmol})$ and diisopropylethylamine $(0.51 \mathrm{ml}, 2.93 \mathrm{mmol})$ in a $2: 1(\mathrm{v} / \mathrm{v})$ mixture of water/tertbutanol $(24 \mathrm{ml})$ was added and the reaction mixture was stirred for a further $24 \mathrm{~h}$ at $353 \mathrm{~K}$. The reaction was quenched with dichloromethane $(c a 40 \mathrm{ml})$ and the heterogeneous mixture was transferred to a separatory funnel. The aqueous

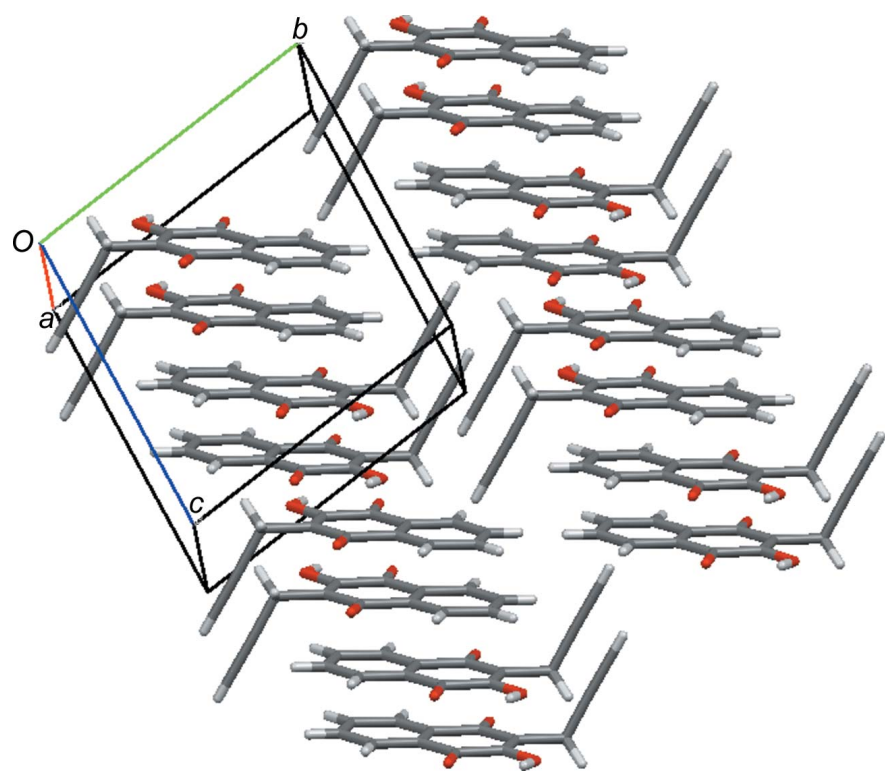

Figure 2

A packing diagram of the title compound. 
<smiles>CC1=CC(=O)C(O)=CC1=O</smiles>

1

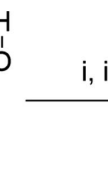

i, ii<smiles>C#CCC1=C(O)C(=O)c2ccccc2C1=O</smiles>

$28 \%$ yield
Figure 3

The synthetic scheme of the title compound, 3; (i) propargyl bromide, sodium iodide and dry acetone, $0.5 \mathrm{~h}$; (ii) diisopropylethylamine and $t$ $\mathrm{BuOH} / \mathrm{H}_{2} \mathrm{O}, 353 \mathrm{~K}, 24 \mathrm{~h}$

phase was separated and the organic layer was extracted with $1 \mathrm{~mol}^{-1}$ hydrochloric acid $(3 \times 40 \mathrm{ml})$ and water $(3 \times 40 \mathrm{ml})$. The organic layer was dried over anhydrous sodium sulfate and concentrated to dryness. The crude red solid product $(0.45 \mathrm{~g})$ was purified by column chromatography (silica) using a 99.5:0.5 ( $v / v)$ mixture of hexane/tert-butanol containing $0.1 \%$ of acetic acid as eluent. Pure title compound was obtained in $28 \%$ yield (0.143 g, m.p. 396.7-397.2 K). Single crystals suitable for X-ray analysis were obtained by slow evaporation of a hexane/tert-butanol solution ( $c a 0.5 \mathrm{mg} \mathrm{ml}^{-1}$ ) at room temperature. The infrared and NMR spectral data and corresponding spectra of $\mathbf{3}$ are available in the supporting information.

\section{Refinement}

Crystal data, data collection and structure refinement details are summarized in Table 2. C-bound $\mathrm{H}$ atoms were placed geometrically $(\mathrm{C}-\mathrm{H}=0.93-0.97 \AA)$ and were refined as riding with $U_{\text {iso }}(\mathrm{H})=1.2 U_{\text {eq }}(\mathrm{C})$. The $\mathrm{O}$-bound $\mathrm{H}$ atom was located in a difference Fourier map and freely refined $[\mathrm{O}-\mathrm{H}=$ 0.89 (3) ̊].

\section{Funding information}

Funding for this research was provided by: Conselho Nacional de Desenvolvimento Científico e Tecnológico (scholarship No. 303901/2017-9); Fundação de Amparo à Pesquisa do Estado de Minas Gerais (grant No. CDS-APQ-02541-15).

\section{References}

Alcantara Emiliano, S., Welma Duarte Silva, S., Alves Pereira, M., R. dos Santos Malta, V. \& Luciano Balliano, T. (2016). Acta Cryst. E72, 188-190.

Ashton, T. M., Fokas, E., Kunz-Schughart, L. A., Folkes, L. K., Anbalagan, S., Huether, M., Kelly, C. J., Pirovano, G., Buffa, F. M., Hammond, E. M., Stratford, M., Muschel, R. J., Higgins, G. S. \& Mckenna, W. G. (2016). Nat. Commun. 7, 12308.

Cirioni, O., Giacometti, A., Balducci, M., Burzacchini, F. \& Scalise, G. (1995). J. Antimicrob. Chemother. 36, 740-741.

Comley, J. C. W., Yeates, C. L. \& Frend, T. J. (1995). J. Antimicrob. Chemother. 39, 806-809.
Table 2

Experimental details.

\begin{tabular}{|c|c|}
\hline \multicolumn{2}{|l|}{ Crystal data } \\
\hline Chemical formula & $\mathrm{C}_{13} \mathrm{H}_{8} \mathrm{O}_{3}$ \\
\hline$M_{\mathrm{r}}$ & 212.19 \\
\hline Crystal system, space group & Triclinic, $P \overline{1}$ \\
\hline Temperature $(\mathrm{K})$ & 293 \\
\hline$a, b, c(\AA)$ & $5.3695(4), 9.5278(8), 10.2972(9)$ \\
\hline$\alpha, \beta, \gamma\left({ }^{\circ}\right)$ & $96.814(7), 93.432(7), 102.977$ (7) \\
\hline$V\left(\AA^{3}\right)$ & $507.68(8)$ \\
\hline$Z$ & 2 \\
\hline Radiation type & Мo $K \alpha$ \\
\hline$\mu\left(\mathrm{mm}^{-1}\right)$ & 0.10 \\
\hline Crystal size $(\mathrm{mm})$ & $0.4 \times 0.2 \times 0.05$ \\
\hline \multicolumn{2}{|l|}{ Data collection } \\
\hline Diffractometer & Rigaku Xcalibur Atlas Gemini ultr \\
\hline Absorption correction & $\begin{array}{l}\text { Multi-scan (CrysAlis PRO; } \\
\quad \text { Rigaku OD, 2015) }\end{array}$ \\
\hline$T_{\min }, T_{\max }$ & $0.720,1.000$ \\
\hline $\begin{array}{l}\text { No. of measured, independent } \\
\quad \text { and observed }[I>2 \sigma(I)] \text { reflections }\end{array}$ & $7946,2508,1563$ \\
\hline$R_{\text {int }}$ & 0.033 \\
\hline$(\sin \theta / \lambda)_{\max }\left(\AA^{-1}\right)$ & 0.695 \\
\hline \multicolumn{2}{|l|}{ Refinement } \\
\hline$R\left[F^{2}>2 \sigma\left(F^{2}\right)\right], w R\left(F^{2}\right), S$ & $0.051,0.147,1.05$ \\
\hline No. of reflections & 2508 \\
\hline No. of parameters & 149 \\
\hline $\mathrm{H}$-atom treatment & $\begin{array}{l}\mathrm{H} \text { atoms treated by a mixture of } \\
\text { independent and constrained }\end{array}$ \\
\hline$\Delta \rho_{\max }, \Delta \rho_{\min }\left(\mathrm{e} \AA^{-3}\right)$ & $0.21,-0.5$ \\
\hline
\end{tabular}

Computer programs: CrysAlis PRO (Rigaku OD, 2015), SHELXT (Sheldrick, 2015a), SHELXL2014 (Sheldrick, 2015b) and OLEX2 (Dolomanov et al., 2009).

Dekkers, J., Kooijman, H., Kroon, J. \& Grech, E. (1996). Acta Cryst. C52, 2896-2899.

Dolomanov, O. V., Bourhis, L. J., Gildea, R. J., Howard, J. A. K. \& Puschmann, H. (2009). J. Appl. Cryst. 42, 339-341.

Fiorillo, M., Lamb, R., Tanowitz, H. B., Mutti, L., Krstic-Demonacos, M., Cappello, A. R., Martinez-Outschoorn, U. E., Sotgia, F. \& Lisanti, M. F. (2016). Oncotarget, 7, 34084-34099.

Groom, C. R., Bruno, I. J., Lightfoot, M. P. \& Ward, S. C. (2016). Acta Cryst. B72, 171-179.

Jordão, A. K., Vargas, M. D., Pinto, A. C., da Silva, F. de C. \& Ferreira, V. F. (2015). RSC Adv. 5, 67909-67943.

Meyer, E. A., Castellano, R. K. \& Diederich, F. (2003). Angew. Chem. 42, 1210-1250

Nasiri, H. R., Madej, M. G., Lancaster, C. R. D., Schwalbe, H. \& Bolte, M. (2006). Acta Cryst. C62, o671-0673.

Nixon, G. L., Moss, D. M., Shone, A. E., Lalloo, D. G., Fisher, N., O’Neill, P. M., Ward, S. A. \& Biagini, G. A. (2013). J. Antimicrob. Chemother. 68, 977-985.

Rigaku OD (2015). CrysAlis PRO. Rigaku Oxford Diffaction, Yarnton, Oxfordshire, England.

Sheldrick, G. M. (2015a). Acta Cryst. A71, 3-8.

Sheldrick, G. M. (2015b). Acta Cryst. C71, 3-8.

Valença, W. O., Baiju, T. V., Brito, F. G., Araujo, M. H., Pessoa, C., Cavalcanti, B. C., Simore, C. A., Jacob, C., Namboothiri, I. N. N. \& da Silva Júnior, E. N. (2017). ChemistrySelect, 2, 4301-4308. 


\section{supporting information}

Acta Cryst. (2018). E74, 1319-1321 [https://doi.org/10.1107/S2056989018011647]

Crystal structure of 2-hydroxy-3-(prop-2-yn-1-yl)naphthalene-1,4-dione

Isidório Raquel Geralda, Ottoni Flaviano Melo, Alves Ricardo José and Speziali Nivaldo Lúcio

Computing details

Data collection: CrysAlis PRO (Rigaku OD, 2015); cell refinement: CrysAlis PRO (Rigaku OD, 2015); data reduction: CrysAlis PRO (Rigaku OD, 2015); program(s) used to solve structure: SHELXT (Sheldrick, 2015a); program(s) used to refine structure: SHELXL2014 (Sheldrick, 2015b); molecular graphics: OLEX2 (Dolomanov et al., 2009); software used to prepare material for publication: OLEX2 (Dolomanov et al., 2009).

2-Hydroxy-3-(prop-2-yn-1-yl)naphthalene-1,4-dione

Crystal data

$\mathrm{C}_{13} \mathrm{H}_{8} \mathrm{O}_{3}$

$M_{r}=212.19$

Triclinic, $P \overline{1}$

$a=5.3695$ (4) $\AA$

$b=9.5278(8) \AA$

$c=10.2972(9) \AA$

$\alpha=96.814(7)^{\circ}$

$\beta=93.432(7)^{\circ}$

$\gamma=102.977(7)^{\circ}$

$V=507.68(8) \AA^{3}$

$Z=2$

$F(000)=220$

$D_{\mathrm{x}}=1.388 \mathrm{Mg} \mathrm{m}^{-3}$

Melting point $=396.8-397.5 \mathrm{~K}$

Mo $K \alpha$ radiation, $\lambda=0.71073 \AA$

Cell parameters from 1721 reflections

$\theta=3.2-28.7^{\circ}$

$\mu=0.10 \mathrm{~mm}^{-1}$

$T=293 \mathrm{~K}$

Prism, colourless

$0.4 \times 0.2 \times 0.05 \mathrm{~mm}$

\section{Data collection}

Rigaku Xcalibur Atlas Gemini ultra diffractometer

Radiation source: fine-focus sealed X-ray tube, Enhance (Mo) X-ray Source

Graphite monochromator

Detector resolution: 10.4186 pixels $\mathrm{mm}^{-1}$

$\omega$ scans

Absorption correction: multi-scan

(CrysAlis PRO; Rigaku OD, 2015)

\section{Refinement}

Refinement on $F^{2}$

Least-squares matrix: full

$R\left[F^{2}>2 \sigma\left(F^{2}\right)\right]=0.051$

$w R\left(F^{2}\right)=0.147$

$S=1.05$

2508 reflections

149 parameters

0 restraints

Primary atom site location: dual

$T_{\min }=0.720, T_{\max }=1.000$

7946 measured reflections

2508 independent reflections

1563 reflections with $I>2 \sigma(I)$

$R_{\text {int }}=0.033$

$\theta_{\text {max }}=29.6^{\circ}, \theta_{\min }=2.8^{\circ}$

$h=-7 \rightarrow 7$

$k=-12 \rightarrow 12$

$l=-13 \rightarrow 14$

Hydrogen site location: mixed

$\mathrm{H}$ atoms treated by a mixture of independent and constrained refinement

$w=1 /\left[\sigma^{2}\left(F_{\mathrm{o}}^{2}\right)+(0.0639 P)^{2}+0.058 P\right]$

where $P=\left(F_{\mathrm{o}}^{2}+2 F_{\mathrm{c}}^{2}\right) / 3$

$(\Delta / \sigma)_{\max }<0.001$

$\Delta \rho_{\max }=0.21 \mathrm{e} \AA^{-3}$

$\Delta \rho_{\min }=-0.5$ e $\AA^{-3}$ 


\section{Special details}

Geometry. All esds (except the esd in the dihedral angle between two 1.s. planes) are estimated using the full covariance matrix. The cell esds are taken into account individually in the estimation of esds in distances, angles and torsion angles; correlations between esds in cell parameters are only used when they are defined by crystal symmetry. An approximate (isotropic) treatment of cell esds is used for estimating esds involving l.s. planes.

Fractional atomic coordinates and isotropic or equivalent isotropic displacement parameters $\left(\hat{A}^{2}\right)$

\begin{tabular}{lllll}
\hline & $x$ & $y$ & $z$ & $U_{\text {iso }} / U_{\text {eq }}$ \\
\hline O3 & $0.6461(3)$ & $0.33269(14)$ & $0.01419(12)$ & $0.0536(4)$ \\
H3 & $0.796(5)$ & $0.389(3)$ & $0.002(3)$ & $0.104(10)^{*}$ \\
O1 & $0.9195(2)$ & $0.59305(13)$ & $0.12532(12)$ & $0.0518(3)$ \\
O2 & $0.0447(3)$ & $0.36386(14)$ & $0.31887(13)$ & $0.0647(4)$ \\
C1 & $0.7232(3)$ & $0.54469(17)$ & $0.17533(15)$ & $0.0388(4)$ \\
C2 & $0.5615(3)$ & $0.39989(17)$ & $0.11881(15)$ & $0.0403(4)$ \\
C3 & $0.3415(3)$ & $0.33844(17)$ & $0.16560(15)$ & $0.0419(4)$ \\
C4 & $0.2483(3)$ & $0.41556(18)$ & $0.27733(16)$ & $0.0431(4)$ \\
C5 & $0.3230(4)$ & $0.6358(2)$ & $0.44404(17)$ & $0.0511(4)$ \\
H5 & 0.1693 & 0.5951 & 0.4767 & $0.061^{*}$ \\
C6 & $0.4664(4)$ & $0.7695(2)$ & $0.50175(19)$ & $0.0586(5)$ \\
H6 & 0.4109 & 0.8182 & 0.5740 & $0.070^{*}$ \\
C7 & $0.6917(4)$ & $0.8311(2)$ & $0.4525(2)$ & $0.0642(6)$ \\
H7 & 0.7871 & 0.9218 & 0.4915 & $0.077^{*}$ \\
C8 & $0.7782(3)$ & $0.7597(2)$ & $0.34557(18)$ & $0.0530(5)$ \\
H8 & 0.9302 & 0.8021 & 0.3123 & $0.064^{*}$ \\
C9 & $0.6345(3)$ & $0.62361(16)$ & $0.28851(15)$ & $0.0389(4)$ \\
C10 & $0.4061(3)$ & $0.56178(17)$ & $0.33796(15)$ & $0.0397(4)$ \\
C11 & $0.1798(4)$ & $0.19019(18)$ & $0.10516(18)$ & $0.0536(5)$ \\
H11A & 0.0054 & 0.1811 & 0.1290 & $0.064^{*}$ \\
H11B & 0.1751 & 0.1833 & 0.0103 & $0.064^{*}$ \\
C12 & $0.2779(4)$ & $0.07067(19)$ & $0.14761(17)$ & $0.0552(5)$ \\
C13 & $0.3566(5)$ & $-0.0258(2)$ & $0.1781(2)$ & $0.0829(7)$ \\
H13 & 0.4196 & -0.1030 & 0.2026 & $0.099^{*}$ \\
\end{tabular}

Atomic displacement parameters $\left(\AA^{2}\right)$

\begin{tabular}{lllllll}
\hline & $U^{11}$ & $U^{22}$ & $U^{33}$ & $U^{12}$ & $U^{13}$ & $U^{23}$ \\
\hline O3 & $0.0570(8)$ & $0.0492(7)$ & $0.0513(7)$ & $0.0084(6)$ & $0.0167(6)$ & $-0.0053(5)$ \\
O1 & $0.0456(7)$ & $0.0531(7)$ & $0.0548(7)$ & $0.0048(6)$ & $0.0176(6)$ & $0.0060(5)$ \\
O2 & $0.0540(8)$ & $0.0635(9)$ & $0.0699(9)$ & $-0.0031(6)$ & $0.0256(7)$ & $0.0043(7)$ \\
C1 & $0.0372(9)$ & $0.0400(8)$ & $0.0405(8)$ & $0.0091(7)$ & $0.0057(7)$ & $0.0094(6)$ \\
C2 & $0.0433(9)$ & $0.0397(9)$ & $0.0391(9)$ & $0.0119(7)$ & $0.0057(7)$ & $0.0047(6)$ \\
C3 & $0.0437(9)$ & $0.0374(8)$ & $0.0430(9)$ & $0.0075(7)$ & $0.0018(7)$ & $0.0040(7)$ \\
C4 & $0.0395(9)$ & $0.0443(9)$ & $0.0453(9)$ & $0.0061(7)$ & $0.0073(7)$ & $0.0110(7)$ \\
C5 & $0.0499(10)$ & $0.0575(11)$ & $0.0468(10)$ & $0.0140(8)$ & $0.0121(8)$ & $0.0047(8)$ \\
C6 & $0.0615(12)$ & $0.0620(12)$ & $0.0503(10)$ & $0.0185(10)$ & $0.0071(9)$ & $-0.0096(8)$ \\
C7 & $0.0615(13)$ & $0.0533(11)$ & $0.0678(13)$ & $0.0076(10)$ & $0.0001(10)$ & $-0.0172(9)$ \\
C8 & $0.0441(10)$ & $0.0488(10)$ & $0.0611(11)$ & $0.0041(8)$ & $0.0066(8)$ & $-0.0004(8)$
\end{tabular}




\begin{tabular}{lllllll} 
C9 & $0.0377(9)$ & $0.0390(8)$ & $0.0402(8)$ & $0.0101(7)$ & $0.0019(7)$ & $0.0046(6)$ \\
C10 & $0.0389(9)$ & $0.0430(9)$ & $0.0380(8)$ & $0.0106(7)$ & $0.0049(7)$ & $0.0055(7)$ \\
C11 & $0.0497(10)$ & $0.0458(10)$ & $0.0582(11)$ & $0.0004(8)$ & $0.0023(9)$ & $0.0006(8)$ \\
C12 & $0.0671(12)$ & $0.0395(10)$ & $0.0515(10)$ & $0.0016(9)$ & $0.0070(9)$ & $-0.0039(8)$ \\
C13 & $0.113(2)$ & $0.0483(12)$ & $0.0862(16)$ & $0.0257(13)$ & $-0.0048(14)$ & $0.0002(11)$ \\
\hline
\end{tabular}

Geometric parameters $\left(\AA,{ }^{\circ}\right)$

\begin{tabular}{|c|c|c|c|}
\hline $\mathrm{O} 3-\mathrm{H} 3$ & $0.88(3)$ & $\mathrm{C} 6-\mathrm{H} 6$ & 0.9300 \\
\hline $\mathrm{O} 3-\mathrm{C} 2$ & $1.3426(19)$ & $\mathrm{C} 6-\mathrm{C} 7$ & $1.375(3)$ \\
\hline $\mathrm{O} 1-\mathrm{C} 1$ & $1.2217(18)$ & $\mathrm{C} 7-\mathrm{H} 7$ & 0.9300 \\
\hline $\mathrm{O} 2-\mathrm{C} 4$ & $1.2189(19)$ & $\mathrm{C} 7-\mathrm{C} 8$ & $1.384(3)$ \\
\hline $\mathrm{C} 1-\mathrm{C} 2$ & $1.485(2)$ & $\mathrm{C} 8-\mathrm{H} 8$ & 0.9300 \\
\hline $\mathrm{C} 1-\mathrm{C} 9$ & $1.473(2)$ & $\mathrm{C} 8-\mathrm{C} 9$ & $1.392(2)$ \\
\hline $\mathrm{C} 2-\mathrm{C} 3$ & $1.340(2)$ & $\mathrm{C} 9-\mathrm{C} 10$ & $1.390(2)$ \\
\hline $\mathrm{C} 3-\mathrm{C} 4$ & $1.465(2)$ & $\mathrm{C} 11-\mathrm{H} 11 \mathrm{~A}$ & 0.9700 \\
\hline $\mathrm{C} 3-\mathrm{C} 11$ & $1.521(2)$ & C11-H11B & 0.9700 \\
\hline $\mathrm{C} 4-\mathrm{C} 10$ & $1.499(2)$ & $\mathrm{C} 11-\mathrm{C} 12$ & $1.458(3)$ \\
\hline $\mathrm{C} 5-\mathrm{H} 5$ & 0.9300 & $\mathrm{C} 12-\mathrm{C} 13$ & $1.161(3)$ \\
\hline $\mathrm{C} 5-\mathrm{C} 6$ & $1.376(3)$ & $\mathrm{C} 13-\mathrm{H} 13$ & 0.9300 \\
\hline $\mathrm{C} 5-\mathrm{C} 10$ & $1.381(2)$ & & \\
\hline $\mathrm{C} 2-\mathrm{O} 3-\mathrm{H} 3$ & $106.5(17)$ & $\mathrm{C} 6-\mathrm{C} 7-\mathrm{C} 8$ & $120.80(18)$ \\
\hline $\mathrm{O} 1-\mathrm{C} 1-\mathrm{C} 2$ & $118.90(14)$ & $\mathrm{C} 8-\mathrm{C} 7-\mathrm{H} 7$ & 119.6 \\
\hline $\mathrm{O} 1-\mathrm{C} 1-\mathrm{C} 9$ & $123.40(15)$ & $\mathrm{C} 7-\mathrm{C} 8-\mathrm{H} 8$ & 120.5 \\
\hline $\mathrm{C} 9-\mathrm{C} 1-\mathrm{C} 2$ & $117.69(14)$ & $\mathrm{C} 7-\mathrm{C} 8-\mathrm{C} 9$ & $119.08(17)$ \\
\hline $\mathrm{O} 3-\mathrm{C} 2-\mathrm{C} 1$ & $115.90(14)$ & $\mathrm{C} 9-\mathrm{C} 8-\mathrm{H} 8$ & 120.5 \\
\hline $\mathrm{C} 3-\mathrm{C} 2-\mathrm{O} 3$ & $120.71(15)$ & $\mathrm{C} 8-\mathrm{C} 9-\mathrm{C} 1$ & $120.23(15)$ \\
\hline $\mathrm{C} 3-\mathrm{C} 2-\mathrm{C} 1$ & $123.38(14)$ & $\mathrm{C} 10-\mathrm{C} 9-\mathrm{C} 1$ & $119.71(14)$ \\
\hline $\mathrm{C} 2-\mathrm{C} 3-\mathrm{C} 4$ & $119.88(15)$ & $\mathrm{C} 10-\mathrm{C} 9-\mathrm{C} 8$ & $120.07(15)$ \\
\hline $\mathrm{C} 2-\mathrm{C} 3-\mathrm{C} 11$ & $122.10(15)$ & $\mathrm{C} 5-\mathrm{C} 10-\mathrm{C} 4$ & $119.53(15)$ \\
\hline $\mathrm{C} 4-\mathrm{C} 3-\mathrm{C} 11$ & $118.01(15)$ & $\mathrm{C} 5-\mathrm{C} 10-\mathrm{C} 9$ & $119.69(15)$ \\
\hline $\mathrm{O} 2-\mathrm{C} 4-\mathrm{C} 3$ & $121.03(16)$ & $\mathrm{C} 9-\mathrm{C} 10-\mathrm{C} 4$ & $120.78(14)$ \\
\hline $\mathrm{O} 2-\mathrm{C} 4-\mathrm{C} 10$ & $120.40(15)$ & $\mathrm{C} 3-\mathrm{C} 11-\mathrm{H} 11 \mathrm{~A}$ & 109.1 \\
\hline $\mathrm{C} 3-\mathrm{C} 4-\mathrm{C} 10$ & $118.56(14)$ & $\mathrm{C} 3-\mathrm{C} 11-\mathrm{H} 11 \mathrm{~B}$ & 109.1 \\
\hline $\mathrm{C} 6-\mathrm{C} 5-\mathrm{H} 5$ & 119.8 & $\mathrm{H} 11 \mathrm{~A}-\mathrm{C} 11-\mathrm{H} 11 \mathrm{~B}$ & 107.8 \\
\hline $\mathrm{C} 6-\mathrm{C} 5-\mathrm{C} 10$ & $120.37(17)$ & $\mathrm{C} 12-\mathrm{C} 11-\mathrm{C} 3$ & $112.70(14)$ \\
\hline $\mathrm{C} 10-\mathrm{C} 5-\mathrm{H} 5$ & 119.8 & $\mathrm{C} 12-\mathrm{C} 11-\mathrm{H} 11 \mathrm{~A}$ & 109.1 \\
\hline $\mathrm{C} 5-\mathrm{C} 6-\mathrm{H} 6$ & 120.0 & $\mathrm{C} 12-\mathrm{C} 11-\mathrm{H} 11 \mathrm{~B}$ & 109.1 \\
\hline $\mathrm{C} 7-\mathrm{C} 6-\mathrm{C} 5$ & $119.98(17)$ & $\mathrm{C} 13-\mathrm{C} 12-\mathrm{C} 11$ & $178.3(2)$ \\
\hline $\mathrm{C} 7-\mathrm{C} 6-\mathrm{H} 6$ & 120.0 & $\mathrm{C} 12-\mathrm{C} 13-\mathrm{H} 13$ & 180.0 \\
\hline $\mathrm{C} 6-\mathrm{C} 7-\mathrm{H} 7$ & 119.6 & & \\
\hline $\mathrm{O} 1-\mathrm{C} 1-\mathrm{C} 2-\mathrm{O} 3$ & $-0.4(2)$ & $\mathrm{C} 4-\mathrm{C} 3-\mathrm{C} 11-\mathrm{C} 12$ & $-100.96(19)$ \\
\hline $\mathrm{O} 1-\mathrm{C} 1-\mathrm{C} 2-\mathrm{C} 3$ & $-179.15(16)$ & $\mathrm{O} 2-\mathrm{C} 4-\mathrm{C} 10-\mathrm{C} 5$ & $2.0(3)$ \\
\hline $\mathrm{C} 9-\mathrm{C} 1-\mathrm{C} 2-\mathrm{O} 3$ & $178.72(14)$ & $\mathrm{O} 2-\mathrm{C} 4-\mathrm{C} 10-\mathrm{C} 9$ & $-177.93(16)$ \\
\hline $\mathrm{C} 9-\mathrm{C} 1-\mathrm{C} 2-\mathrm{C} 3$ & $-0.1(2)$ & $\mathrm{C} 3-\mathrm{C} 4-\mathrm{C} 10-\mathrm{C} 5$ & $-179.45(16)$ \\
\hline $\mathrm{O} 1-\mathrm{C} 1-\mathrm{C} 9-\mathrm{C} 8$ & $-1.0(2)$ & $\mathrm{C} 3-\mathrm{C} 4-\mathrm{C} 10-\mathrm{C} 9$ & $0.7(2)$ \\
\hline
\end{tabular}




$\begin{array}{llll}\mathrm{O} 1-\mathrm{C} 1-\mathrm{C} 9-\mathrm{C} 10 & 178.81(15) & \mathrm{C} 10-\mathrm{C} 5-\mathrm{C} 6-\mathrm{C} 7 & 1.0(3) \\ \mathrm{C} 2-\mathrm{C} 1-\mathrm{C} 9-\mathrm{C} 8 & 179.98(14) & \mathrm{C} 6-\mathrm{C} 5-\mathrm{C} 10-\mathrm{C} 4 & 179.40(17) \\ \mathrm{C} 2-\mathrm{C} 1-\mathrm{C} 9-\mathrm{C} 10 & -0.2(2) & \mathrm{C} 6-\mathrm{C} 5-\mathrm{C} 10-\mathrm{C} 9 & -0.7(3) \\ \mathrm{O} 3-\mathrm{C} 2-\mathrm{C} 3-\mathrm{C} 4 & -178.09(15) & \mathrm{C} 5-\mathrm{C} 6-\mathrm{C} 7-\mathrm{C} 8 & -0.4(3) \\ \mathrm{O} 3-\mathrm{C} 2-\mathrm{C} 3-\mathrm{C} 11 & 1.0(2) & \mathrm{C} 6-\mathrm{C} 7-\mathrm{C} 8-\mathrm{C} 9 & -179.48(16) \\ \mathrm{C} 1-\mathrm{C} 2-\mathrm{C} 3-\mathrm{C} 4 & 0.7(2) & \mathrm{C} 7-\mathrm{C} 8-\mathrm{C} 9-\mathrm{C} 1 & 0.7(3) \\ \mathrm{C} 1-\mathrm{C} 2-\mathrm{C} 3-\mathrm{C} 11 & 179.77(15) & \mathrm{C} 7-\mathrm{C} 8-\mathrm{C} 9-\mathrm{C} 10 & -0.1(2) \\ \mathrm{C} 2-\mathrm{C} 3-\mathrm{C} 4-\mathrm{O} 2 & 177.64(16) & \mathrm{C} 1-\mathrm{C} 9-\mathrm{C} 10-\mathrm{C} 4 & 179.00(16) \\ \mathrm{C} 2-\mathrm{C} 3-\mathrm{C} 4-\mathrm{C} 10 & -0.9(2) & \mathrm{C} 1-\mathrm{C} 9-\mathrm{C} 10-\mathrm{C} 5 & -0.2(2) \\ \mathrm{C} 11-\mathrm{C} 3-\mathrm{C} 4-\mathrm{O} 2 & -1.5(2) & \mathrm{C} 8-\mathrm{C} 9-\mathrm{C} 10-\mathrm{C} 4 & \mathrm{C} 8-\mathrm{C} 9-\mathrm{C} 10-\mathrm{C} 5 \\ \mathrm{C} 11-\mathrm{C} 3-\mathrm{C} 4-\mathrm{C} 10 & 179.92(15) & & \end{array}$

Hydrogen-bond geometry $\left(\AA,{ }^{\circ}\right)$

\begin{tabular}{lllll}
\hline$D-\mathrm{H} \cdots A$ & $D-\mathrm{H}$ & $\mathrm{H} \cdots A$ & $D \cdots A$ & $D-\mathrm{H} \cdots A$ \\
\hline $\mathrm{O} 3-\mathrm{H} 3 \cdots \mathrm{O} 1^{\mathrm{i}}$ & $0.89(3)$ & $2.06(3)$ & $2.8118(19)$ & $142(3)$ \\
$\mathrm{C} 5-\mathrm{H} 5 \cdots \mathrm{O} 2^{\mathrm{ii}}$ & 0.93 & 2.49 & $3.231(2)$ & 137 \\
\hline
\end{tabular}

Symmetry codes: (i) $-x+2,-y+1,-z$; (ii) $-x,-y+1,-z+1$. 